\title{
Clinical forms of Trypanosoma cruzi infected individuals in the chronic phase of Chagas disease in Puebla, Mexico
}

\author{
María del Carmen Sánchez-Guillén, Aurelio López-Colombo*, \\ Guillermo Ordóñez-Toquero**, Isidoro Gomez-Albino***, Judith Ramos-Jimenez, \\ Enrique Torres-Rasgado/ ${ }^{++}$, Hilda Salgado-Rosas/ ${ }^{++}$, Mónica Romero-Díaz, \\ Patricia Pulido-Pérez, Ricardo Pérez-Fuentes/****/+
}

Laboratorio de Investigación en Fisiopatología de Enfermedades Crónicas, Centro de Investigación Biomédica de Oriente, IMSS, Puebla, México *Servicio de Gastroenterología **Servicio de Cardiología ***Servicio de Radiología, Hospital de Especialidades,

Centro Médico Nacional, IMSS Puebla, México ****Facultad de Medicina, Benemérita Universidad Autónoma de Puebla, Puebla, México

In Mexico, despite the relatively high seroprevalence of Trypanosoma cruzi infection in humans in some areas, reported morbidity of Chagas disease is not clear. We determined clinical stage in 71 individuals seropositive to T. cruzi in the state of Puebla, Mexico, an area endemic for Chagas disease with a reported seroprevalence of $7.7 \%$. Diagnosis of Chagas disease was made by two standardized serological tests (ELISA, IHA). Individuals were stratified according to clinical studies. All patients were submitted to EKG, barium swallow, and barium enema. Groups were identified as indeterminate form (IF) asymptomatic individuals without evidence of abnormalities ( $\mathrm{n}=34$ cases); those with gastrointestinal alterations (12 patients) including symptoms of abnormal relaxation of the lower esophageal sphincter and absent peristalsis in the esophageal body, grade I megaesophagus, and/or megacolon; patients with clinical manifestations and documented changes of chronic Chagas heart disease who were subdivided as follows: mild (8 patients) - mild electrocardiographic changes of ventricular repolarization, sinus bradychardia); moderate (6 patients) - left bundle branch block, right bundle branch block associated with left anterior fascicular block); severe (8 patients) - signs of cardiomegaly, dilated cardiomyopathy); and the associated form (3 cases) that included presence of both cardiomyopathy and megaesophagus. These data highlight the importance of accurate evaluation of the prevalence and clinical course of Chagas disease in endemic and non-endemic areas of Mexico.

Key words: clinical forms - Chagas disease - seropositive - Trypanosoma cruzi - cardiomyopathy - indeterminate form

Chagas disease is caused by the protozoan parasite Trypanosoma cruzi and is now ranked as the most serious parasitic disease of the Americas with an economic impact far outranking the combined effects of other parasitic diseases such as malaria, schistosomiasis and leishmaniasis (Dias \& Schofield 1999).

Current World Health Organization estimates indicate that 16-18 million people are infected with T. cruzi (Moncayo 1992), 60\% live in urban areas, and 50\% are in a clinically latent period. Peru, Colombia, and part of Bolivia still have no control programs, as in Mexico, and new cases are expected year by year (Moncayo 2003). Therefore, because many infected people are potential blood donors, one can expect that 100 million additional persons are at risk of contracting the disease (MoraesSouza \& Bordin 1996, OPAS 1998, Dias \& Schofield 1999).

The human form of the disease has a variable clinical presentation. Following parasite infection, there is a short

\footnotetext{
Financial support: Fosiza-Conacyt project 9980802019

${ }^{+}$Corresponding author: rycardoperez@yahoo.com.mx

${ }^{++}$Fellowship from Conacyt

Received 4 April 2006

Accepted 13 September 2006
}

acute phase characterized by an abundant parasitemia that can be seen relatively easily by direct blood examination. Frequently, very mild and nonspecific symptoms make recognition of the contagion difficult. Without specific treatment, about $5-10 \%$ of symptomatic patients may die due to encephalomyelitis or severe cardiac failure (Moncayo 1992). However, in the vast majority of patients this acute phase of the disease goes unrecognized because of the scarcity or absence of clinical manifestations.

After 2-4 months the acute clinical manifestations disappear and the disease enters the chronic phase, generally starting with a long, asymptomatic clinical latency, which lasts 10-30 years or throughout life. In this phase, up to $30 \%$ of infected individuals develop at least one of the clinical forms of the disease: cardiomyopathy, and/ or megagastrointestinal syndromes, lacking effective treatment (Prata 2001). From 50 to $70 \%$ of individuals remain asymptomatic and do not exhibit abnormalities in clinical studies (Tay et al. 1992). Chagas disease is confined to the Americas and ranks 4th according to World Health Organization (WHO 2002) priority diseases. It is the most frequent cause of cardiopathy in endemic areas, especially in rural areas in the Americas (UNDP 1990).

In Mexico, despite endemicity of $T$. cruzi parasites in vectors and reservoirs and the relatively high 
prevalence in humans, morbidity due to Chagas disease is not clear.

In this work we report the results of the clinical characteristics in seropositive individuals to T. cruzi from the state of Puebla, Mexico, an area endemic for Chagas disease. Diagnosis of Chagas disease was made by two standardized serological tests using one autochthonous antigen from $T$. cruzi parasites by xenodiagnostics from a human host from the state of Puebla, Mexico and genetically characterized as HUM/ME/1997/MEX/RyCH1 (T. cruzi I).

\section{MATERIALS AND METHODS}

Study area - The study was conducted in the state of Puebla (Fig. 1), which is located in the Southeast region of Mexico in a valley $1800 \mathrm{~m}$ above sea level and with a warm climate (average annual temperature $22^{\circ} \mathrm{C}$ ). The population is 5.1 million persons, with $60 \%$ living in rural and suburban areas under ecological and socioeconomic conditions appropriate for the transmission of T. cruzi parasites. In this endemic area for Chagas disease, reported seroprevalence is $7.7 \%$ (Sanchez-Guillén et al. 2002).

Subjects - We studied the seroprevalence in 2140 random blood samples from blood donors, evaluated for anti-T. cruzi antibodies, from the seven areas of the state of Puebla. We selected 166 individuals who were seropositive to both assays, i.e., enzyme-linked immunosorbent assay (ELISA) and indirect hemagglutination (IHA) and who were considered carriers of T. cruzi antibodies. Seventeen (10\%) were included according to selection criteria (Sánchez-Guillén et al. 2002) and 54 asymptomatic and symptomatic patients with clinical data associated with chronic Chagas disease and who were seropositive to both assays (ELISA and IHA). Subjects were prospectively recruited from the Gastroenterology and Cardiology Services at Centro
Medico Nacional Manuel Avila Camacho, Instituto Mexicano del Seguro Social (IMSS), Puebla, Mexico. Among seropositive individuals, those with negative (1 of 2 tests) serology or seropositive to HIV (two individuals), VDRL, Brucella abortus (one individual), hepatitis B surface antigen (HBs Ag), hepatitis B core antigen ( $\mathrm{HBc} \mathrm{Ag}$ ) and hepatitis $\mathrm{C}$ virus ( $\mathrm{HCV}$ ) (two individuals) tests were excluded. Other exclusion criteria were diagnosis of other chronic diseases or presence of contradictory findings from repeated examinations, refusal to participate (50 individuals) or due to difficulties as a result of rural location (94 individuals).

Confirmatory diagnosis of Chagas disease was made in all patients on the basis of detection of circulating antibodies against $T$. cruzi antigens by two standardized serological tests according to the WHO (2002).

Clinical studies - All individuals enrolled were evaluated by a physician who administered an epidemiological questionnaire. Subjects were evaluated on the basis of previous illnesses and clinical history including sign and symptoms such as chronic diseases, dyspnea, cardiopathy, lower extremity edema, odinophagia, dysphagia, pyrosis, hyposialorrea, and constipation. Clinical studies included 12-lead electrocardiogram (ECG), echocardiogram (EchoCG), and a heart roentgenogram (2-m distance) to determine cardiothoracic index. Esophageal manometry and radiological imaging of the esophagus and colon were obtained using conventional techniques. A barium contrast fluoroscopic esophagram was done with oblique and posteroanterior views in all patients. All patients were instructed to use an enema prior to the colon study. Barium was administered rectally. The studies were made by a radiologist and a cardiologist according to the criteria of the Cardiology and Radiology Services of Centro Médico Nacional Manuel Ávila Camacho.

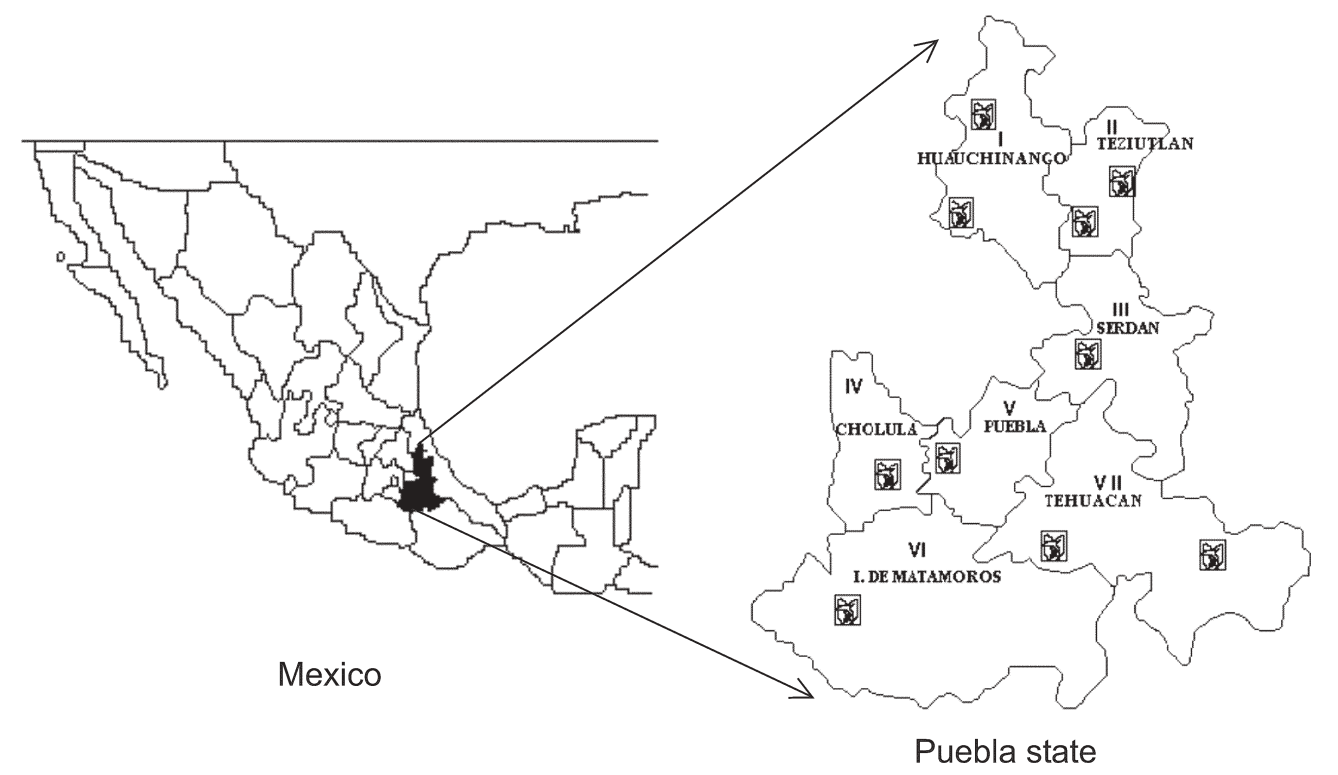

Fig. 1: geographical localization of the studied patients. 
Informed consent was obtained from each subject and the study protocol was approved by the Hospital Research Committee from the state of Puebla.

Serum samples -To minimize alterations of blood components and to standardize samples, venous blood was obtained in the morning after an overnight fast and allowed to clot at room temperature for $2 \mathrm{~h}$. Serum was separated by centrifugation $(1200 \times \mathrm{g}$ for $10 \mathrm{~min})$, frozen at $-20^{\circ} \mathrm{C}$, and stored at $-70^{\circ} \mathrm{C}$ until use.

Antigen - Total antigen extracts from T. cruzi parasites were obtained by xenodiagnostics from a human host from the state of Puebla, Mexico. Isolated parasites were characterized by multilocus enzyme electrophoresis (MLEE), random amplified polymorphic DNA (RAPDs), and biodemes such as HUM/ME/1997/ MEX/RyCH1 (Perez-Fuentes et al. 2003). Parasites were cultured and propagated in liver infusion tryptose medium supplemented with $10 \%$ fetal calf serum. In brief, parasites (epimastigotes) were grown at logarithmic phase, harvested, sonicated in the presence of protease inhibitors, and centrifuged at $10,000 \times \mathrm{g}$ for $30 \mathrm{~min}$ at $4^{\circ} \mathrm{C}$. The resulting supernatant was used as crude antigen extract. Protein concentrations quantified by the Lowry method were adjusted to $1 \mathrm{mg} / \mathrm{ml}$ and stored at $-70^{\circ} \mathrm{C}$. The same strain was used for ELISA and for IHA tests.

\section{Serologic analysis}

ELISA test - Briefly, polystyrene plates (Dynatech Corp., Chantilly, VA) were sensitized with the crude antigen extract of epimastigotes at a concentration of 100 $\mu \mathrm{g} / \mathrm{ml}$ in carbonate buffer, $\mathrm{pH} 9.5$, and blocked with fetal bovine serum at $1 \%$ in PBS-Tween $20(0.01 \%)$. Blood donor and control sera were diluted 1:100, incubated for $1 \mathrm{~h}$, and washed with PBS. Conjugated human anti-IgG coupled to horseradish peroxidase was used. Colorimetric reaction was developed with orthophenylenediamine and hydrogen peroxide, and the reaction was stopped and read at $490 \mathrm{~nm}$ in an ELISA reader.
IHA technique - Sensitization of red blood red cells was required for this assay. Ram erythrocytes were used at a concentration of $2.5 \%$ in phosphate buffer $(\mathrm{pH} 7.2)$ mixed with tannic acid at a dilution of $1: 60,000$, sensitized with $T$. cruzi antigen at $37^{\circ} \mathrm{C}$ for $20 \mathrm{~min}$, washed with PBS ( $\mathrm{pH}$ 7.2) and adjusted at a concentration of 0.2 $\mathrm{mg} / \mathrm{ml}$. Patients' sera at dilutions 1:8, 1:32, and 1:64 were incubated for $2 \mathrm{~h}$ at room temperature. Antibody identification was determined by the presence of agglutination at the bottom of the plates. A titer of 1:8 was considered as infection and 1:32 as confirmatory. All samples were analyzed in triplicate and repeated twice.

\section{RESULTS}

Criteria and clinical grouping of patients - The study population consisted of 71 subjects (39 men and 32 women, mean age $39.39 \pm 15.98$ years, range: $12-90$ years), seropositive to $T$. cruzi antigens with both ELISA and IHA assays, and who fulfilled the selection criteria. Epidemiological and demographic characteristic of asymptomatic subjects and patients including age, gender and serologic data are shown in Tables I and II.

All selected subjects were seronegative to HIV,

TABLE I

Epidemiological characteristics

\begin{tabular}{lc}
\hline Parameter & Positive (\%) \\
\hline Knowledge of Chagas disease & 10 \\
Knowledge of triatomines & 20 \\
Place of residence & \\
$\quad$ Rural & $20 \%$ \\
Suburban & $65 \%$ \\
$\quad$ Urban & $15 \%$ \\
Schooling (years) & $6 \pm 2$ \\
\hline
\end{tabular}

Rural: lack of sanitary services and presence of poor housing along with coexistence with domestic and peridomestic animals. Suburban: poor housing situated in peripheral area of big cities with lack of sanitary services. Urban: individuals living in an area with total sanitary services.

TABLE II

Demographic characteristics

\begin{tabular}{|c|c|c|c|c|}
\hline \multirow[t]{2}{*}{ Clinical group } & \multicolumn{3}{|c|}{ Gender } & \multirow{2}{*}{$\begin{array}{c}\text { Age (years) } \\
\mu \mathrm{m} \pm \mathrm{SD} \text { (range) }\end{array}$} \\
\hline & Male & Female & Total & \\
\hline Indeterminate form $^{a}$ & 17 & 17 & 34 & $\begin{array}{c}37.7 \pm 18.16 \\
(12-90)\end{array}$ \\
\hline Gastrointestinal alterations ${ }^{b}$ & 7 & 5 & 12 & $\begin{array}{c}47.66 \pm 22.64 \\
(20-84)\end{array}$ \\
\hline Cardiac alterations ${ }^{c}$ & 14 & 8 & 22 & $\begin{array}{c}39 \pm 13.48 \\
(21-60)\end{array}$ \\
\hline Combined $^{d}$ & 1 & 2 & 3 & $\begin{array}{c}48.33 \pm 23.67 \\
(21-62)\end{array}$ \\
\hline
\end{tabular}

Seropositive subjects to Trypanosoma cruzi antigens were stratified according to manometric, electrocardiographic and echocardiographic criteria. $a$ : indeterminate form: seropositive individuals to anti-T. cruzi without clinical alterations; $b$ : patients with documented changes by manometric criteria and colon changes by X-ray with contrast media; $c$ : patients with electrocardiographic and/or echocardiographic changes; $d$ : patients with gastrointestinal and cardiac alterations. 


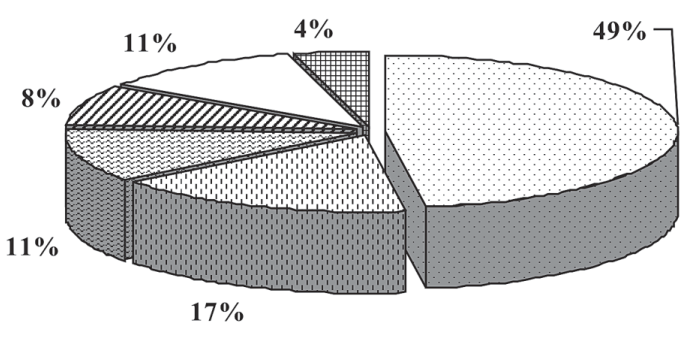

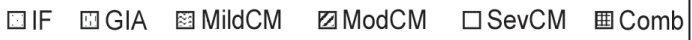

Fig. 2: clinical classification of 71 study individuals. IF: indeterminate form; GIA: gastrointestinal alterations; MildCM: mild cardiomyopathy; ModCM: moderate cardiomyopathy; SevCM: severe cardiomyopathy; Comb: chronic Chagas disease with gastrointestinal and cardiac alterations.

VDRL, Brucella abortus, $\mathrm{HBs} \mathrm{Ag}, \mathrm{HBc} \mathrm{Ag}$ and $\mathrm{HCV}$ tests and were classified according to clinical findings (Fig. 2).

The group of 34 seropositive individuals who were free of overt symptoms or symptoms of cardiac or megagastrointestinal disease and without evidence of abnormalities or alterations in clinical studies was classified as indeterminate form (IF).

Gastrointestinal symptoms (dysphagia, constipation, abdominal pain) and documented abnormalities of the digestive tract were found in 12 seropositive patients and were classified as gastrointestinal group (GG). Documented changes by manometry including abnormal relaxation of the lower esophageal sphincter (AR-LES) and absence of peristalsis in the esophageal body were observed in four patients. Radiologic imaging of the gastrointestinal tract showed Group 2 megaesophagus with delayed emptying time (> $60 \mathrm{~s}$ ) and an enlargement up to $4.5 \mathrm{~cm}$ of the esophagus diameter in four patients and megacolon with an enlargement of the esophagus diameter from 5 to $9.5 \mathrm{~cm}$ in four patients (Group 2 megaesophagus) (Table III). Gastroesophageal reflux was found in two patients with Group 1 megaesophagus.

Patients with clinical manifestation of chronic Chagas cardiomyopathy were likewise subdivided into mild, moderate, and severe categories: symptomatic and with documented changes on an ECG and chest X-ray or EchoCG consistent with cardiomyopathy as determined by a cardiologist or by X-rays.

The eight cases with criteria for mild cardiomyopathy were without electrocardiographic changes or mild changes such as ventricular repolarization or sinus bradychardia. Among these patients, three showed grade I dyspnea and the remaining five were normal. Echocardiograms were normal. Criteria in the group considered to have moderate cardiomyopathy included six patients with ECG changes, indicative of heart damage of chagasic etiology: incomplete left bundle branch block (ILBBB) (one case), complete left bundle branch block (CLBBB) (one case), and complete right bundle branch block (CRBBB) associated with left anterior fascicular block (three cases). Among these patients, three showed grade II dyspnea, two showed grade I dyspnea, and one was normal. Echo-cardiograms remained normal. The severe cardiomyopathy group (eight patients) was classified based on the presence of cardiomegaly, indicative of dilated cardiomyopathy and clinical symptoms: all presented grade III dyspnea and four patients suffered from syncope). Echocardiogram showed dilated cardiomyopathy in all cases. In addition to cardiomegaly, five of eight cases presented with complete RBBB and three with complete atrioventricular block (A-VB). The clinical category, referred to as the combined form, was characterized by the presence of both cardiomyopathy

TABLE III

Clinical groups according to clinical, electrocardiographic, echocardiographic, manometric, and X-ray studies

\begin{tabular}{lrl}
\hline Clinical groups & $n$ & Clinical criteria \\
\hline Indeterminate form & 34 & Normal heart size \\
& & Normal electrocardiogram \\
& Normal esophageal manometry \\
& & Normal barium enema \\
Gastrointestinal alterations & 12 & Gastrointestinal symptoms: dysphagia, constipation, retrosternal pain \\
& 4 & AR-LES, GER \\
& 4 & Achalasia megaesophagus \\
& 4 & Group 1abnormal relaxation of colon plus Group 2 megaesophagus
\end{tabular}

Clinical forms of cardiopathy

$\begin{array}{lll}\text { Mild } & 8 & \text { Conduction alterations } \\ \text { Moderate } & 6 & \text { CRBBB (4), CLBBB (1), ILBBB (1) } \\ \text { Severe } & 8 & \text { Dilated cardiomyopathy5 CRBBB, 3 A-VB } \\ \text { Combination form } & 3 & \text { Cardiomyopathy and megaesophageal syndromes }\end{array}$

AR-LES: abnormal relaxation of lower esophageal sphincter by esophageal manometry; GER: gastroesophageal reflux; CRBBB: complete right bundle branch block; CLBBB: complete left bundle branch block; ILBBB: incomplete left bundle branch block; A-VB: atrioventricular block. 
and megaesophagus (three cases). Clinical characteristics are shown in Table II.

\section{DISCUSSION}

In Mexico, information on the prevalence of Chagas disease is scarce and the public health importance of the disease is debated (Velasco \& Guzman 1986, Tay et al. 1992). A number of triatomine vectors and wild and domestic reservoirs have been identified (Cruz-Reyes et al. 2006). Seroprevalence of T. cruzi infection in the reservoir has been reported to vary between $8 \%$ and $62 \%$ according to species and regions (Cruz-Reyes et al. 2006). With respect to human infection, the National Seroepidemio-logical Survey (NSS) (Velasco et al. 1992) carried out by the official Secretaría de Salud (SSa) showed a prevalence of $1.6 \%$, whereas independent studies carried out mainly in rural areas have shown that between 20 and $30 \%$ of the population was infected by the pathogen (Monteon \& Reyes-Lopez 1989, Tay et al. 1992). Moreover, positive blood donors have been detected at percentages of $0.2-17 \%$ of infected persons (Ramos-Echevarria et al. 1993, Guzman-Bracho et al. 1998, Rangel et al. 1998, Sánchez-Guillén et al. 2002, Hernandez-Becerril et al. 2005, Monteon et al. 2005, Kirchhoff et al. 2006).

The seroprevalence found in blood donors from the state of Puebla (7.7\%) is relatively high and comparable to those found in endemic states of others countries. These findings are in sharp contrast with the National Seroepi-demiological Survey in Mexico carried out in 1987 and published in 1992, which showed a low prevalence of $1.6 \%$. These differences may, in part, be attributed to the fact that this last study was performed with statistics specifically designed for the characterization of transmissible urban diseases such as AIDS and hepatitis, but not for transmissible rural diseases such as Chagas disease (Ve-lasco et al. 1992). Additionally, our findings (7.7\%) contrast with the more recent study carried out by the official SSa showing only $1.5 \%$ of national prevalence and specifically $1.8 \%$ from the state of Puebla (Guzmán-Bracho et al. 1998). In this case, the discrepancy could be explained in part by the serologic tests employed (both ELISA and IHA assays), the strain of the parasite used in the antigen preparation (autochthonous antigen), and mainly by the size (random sample) and characteristic of the analyzed population. Recently, Monteon et al. (2005) reported a seroprevalence from Puebla of $1.24 \%$ using previously validated procedures.

The antibody-based immunoassay is a relevant diagnostic tool because of its high sensitivity. In the particular case of Chagas disease, no serologic "gold" standard exists because detection of $T$. cruzi-specific antibodies depends on many factors. Nevertheless, IFA is the most commonly used serologic test for Chagas disease and, as a result, is widely accepted as the gold standard (Ferreira \& Moraes de Avila 1995). Recent reports show that in-house IFA and in-house ELISA were highly concordant (Oelemann et al. 1998). As suggested by Organización Panamericana de la Salud (OPAS), IHA is a screening technique whereas ELISA or IFA is for confirmatory diagnosis with a higher specificity than IHA. In our report, in complete agreement with the OPAS recommendations that mandatory serology must be implemented in all countries where T. cruzi is endemic by using at least two serological tests (OPAS 1998), we used two independent techniques, i.e., in-house IHA and in-house ELISA with the same antigen. The latter assay presented a higher specificity for detection of Chagas disease (Pérez-Fuentes et al. 1998).

Our findings can be explained in part by the type of antigen we used in serologic tests. Further studies have suggested that the specificity of different methods depends on the selection of an adequate antigen (Mendes et al. 1997), considering that T. cruzi is polymorphic, and that different parasite strains circulate in different areas (Dias 1992). Genetic characterization of T. cruzi parasites has shown that Mexican stocks belonging to $T$. cruzi I and II are closely related (Bosseno et al. 2002). In Mexico, most studies have been performed using $T$. cruzi antigens extracted from strains originating from other geographical origins and showing different genotypes (Bucio et al. 1999).

We have shown previously that assays utilizing commercial antigens from other countries are less sensitive than using T. cruzi autochthonous antigen in the diagnosis of Chagas diseases (Pérez-Fuentes et al. 1998). This finding was recently demonstrated by other authors (Sánchez et al. 2001).

The specificity of our analysis was to use autochthonous antigens from local T. cruzi strains isolated in the same area and characterized as T. cruzi I. These antigens could naturally reflect locally adapted host immune response against circulating $T$. cruzi clones in the area.

Together these vectors, reservoir, and seroepidemiological studies clearly demonstrate that $T$. cruzi infection is a zoonosis endemic in many regions of Mexico and that there are various geographical regions with a relatively high seroprevalence of human $T$. cruzi infection, e.g., Costa Chica Guerrero, Oaxaca and Chiapas (Guzman-Bracho et al. 1998). However, because the first cases of human infection were identified in 1940 in Mexico by Mazzotti, and until 1991 only about 300 clinical cases of chronic Chagas disease had been officially reported (Dumonteil 1999), it is difficult in Mexico to document the incidence of human infection and diseases.

In this work we report the results of our investigation in a group of asymptomatic carriers of T. cruzi antibodies identified from a previous study (Sanchez-Guillen et al. 2002) and asymptomatic and symptomatic patients with clinical data associated with chronic Chagas disease. These subjects were prospectively recruited from the Gastroenterology and Cardiology Services at Centro Medico Nacional Manuel Avila Camacho, IMSS, state of Puebla, Mexico and were evaluated clinically for presence, absence and severity of chronic Chagas disease. The human disease has a variable clinical presentation.

Geographic variations in the prevalence of clinical forms and morbidity of Chagas disease have been reported. In Brazil, the asymptomatic or indeterminate form is the most common (60-70\%), followed by the 
cardiac and digestive forms (20-30 and 8-10\%, respectively). In Brazil, approximately $50 \%$ of the cases have megaesophagus with megacolon and 33\% present chagasic cardiopathy associated. However, in central Brazil and in Chile, the digestive form of Chagas disease predominates, whereas it is practically non-existent in Venezuela and Central America (Luquetti et al. 1986, Dias 1992).

In Mexico, scarce epidemiological data are available about the incidence of chronic chagasic disease (cardiomyopathy and megasyndromes) (Barrera-Perez et al. 1992). Some isolated cases have been described in different states (Huante-Magaña et al. 1990, Perez-Fuentes et al. 1998, Revollo et al. 1998). In this region, cardiac problems due to Chagas disease are reported to be more prevalent than gastrointestinal disease (MendozaGonzalez et al. 1995). In this work, all individuals resided in the study region. Among these, $\sim 48 \%$ of asymptomatic individuals mainly from the seroprevalence study were diagnosed with the indeterminate form without evidence of abnormalities, and $40 \%$ of symptomatic patients from Cardiology Services at Centro Medico Nacional Manuel Avila Ca-macho, IMSS presented clinical manifestations and documented changes of chagasic cardiomyopathy. They were then subdivided into the following categories: mild (8 cases) - without electrocardiographic changes or mild changes such as changes of ventricular repolarization or sinus bradycardia observed in those individuals $<35$ years old; moderate (6 cases) - with electrocardiographic changes indicative of heart damage of chagasic etiology: LBBB, RBBB, associated with left anterior fascicular block; and severe form (8 cases) with signs of cardiomegaly: dilated cardiomyopathy was found more often in cases $>45$ years old associated with clinical symptoms. Frequency of chronic Chagas heart disease found in this work is comparable to the highest rates reported in other studies performed in South America (Maguire et al. 1982, Acquatella et al. 1987).

Despite the fact that most individuals infected with T. cruzi remain in the chronic indeterminate phase of infection (UNDP 1990), it is important to consider that asymptomatic subjects with Chagas disease may have cardiac and esophageal alterations detected only by imaging studies (Cortes et al. 1985, Storino \& Milei 1986).

Individuals in this study were evaluated using esophageal manometry and radiological imaging of the esophagus and colon. Interestingly, we found that asymptomatic individuals $(<35$ years old) and those without evidence of abnormalities had abnormal relaxation of the LES and absent peristalsis in the esophageal body (four patients), whereas eight patients presented grade I megaesophagus. Megacolon was determined in four patients. The combined form, characterized by presence of both cardiomyopathy and megaesophagus, was identified in only three cases.

In Mexico, infection by T. cruzi is mainly silent, as in most Latin American countries. The fact that few chronic cases are reported in Mexico despite endemicity of $T$. cruzi parasites in vectors and reservoirs and the relatively high seroprevalence in humans may be due to a low virulence of the Mexican strains of T. cruzi. Because we know that $T$. cruzi, the agent of Chagas disease, exhibits considerable biological variability, it has been suggested that parasite strains isolated from one endemic area with similar biological and isoenzymatic patterns could correspond to distinct epidemiological and clinical pictures.

Our findings can be partly explained by the type of parasite strains, considering that $T$. cruzi is polymorphic and that different parasite strains circulate in different areas (Dias 1992). Genetic characterization of T. cruzi parasites has shown that Mexican stocks belong mainly to $T$. cruzi I and are closely related to each other (Bosseno et al. 2002). We characterized the genotype by MLEE and pathological profile (biodemes) of T. cruzi stocks from parasites isolated in the same area. Parasites were isolated from triatomine vectors, domestic reservoirs and human host and characterized as T. cruzi I by MLEE as belonging to discrete typing units 1 (DTU1) and biodeme type III (Perez-Fuentes et al., in press).

In conclusion, we found significant morbidity in chronic Chagas disease in an endemic area to Chagas disease, with a reported seroprevalence of $7.7 \%$. Our findings suggest that infection, cardiac morbidity, and gastrointestinal pathology may be substantially higher in some endemic areas in Mexico.

Data presented in this work are relevant because they clearly establish the prevalence of clinical forms of chronic Chagas disease in T. cruzi-seropositive individuals and provide information in Mexico, where data regarding the importance of Chagas disease are either limited or underestimated.

\section{ACKNOWLEDGMENTS}

To the medical personnel of the Cardiology, Gastroenterology, and Radiology Services of Centro Medico Nacional Manuel Avila Camacho, IMSS, Puebla, Mexico for conducting the clinical studies.

\section{REFERENCES}

Acquatella H, Catalioti F, Gomez-Mancebo JR, Davalos V, Villalobos L 1987. Long term control of Chagas disease in Venezuela: effects on serologic findings, electrocardiographic abnormalities, and clinical outcome. Circulation 76: 556-562.

Barrera-Pérez MA, Rodríguez-Felix ME, Guzmán-Marín E, Zavala-Velazquez JE 1992. Prevalencia de la enfermedad de Chagas en el estado de Yucatán. Rev Biomed 3: 133-139.

Bosseno MF, Barnabé C, Magallón-Gastélum E, Lozano-Kasten F, Ramsey J, Espinoza B, Breniére SF 2002. Predominance of Trypanosoma cruzi lineage I in Mexico. J Clin Microbiol 40: 627-632.

Bucio MI, Cabrera M, Segura EL, Zenteno E, Salazar-Schettino M 1999. Identification of immunodominant antigens in Mexican strains of Trypanosoma cruzi. Immunol Invest 28: 257-268.

Cortes JM, Velasco CO, Labastida MH, Melchor AH, Duarte N, De-Torre R 1985. La enfermedad de Chagas en Santiago Yosotiche, Oaxaca, Mexico. Salud Publica Mex 27: 60-65.

Cruz-Reyes A, Pickering-Lopez JM 2006.Chagas disease in Mexico: an analysis of geographical distribution during the 
past 76 years - A review. Mem Inst Oswaldo Cruz 101: 345-354.

Dias JC 1992. Epidemiology of Chagas disease. In S Wendel, Z Brener, ME Camargo, A Rassi (eds), Chagas Disease (American Trypanosomiasis): Its Impact on Transfusion and Clinical Medicine, International Society of Blood Transfusion, São Paulo, p. 49-80.

Dias J, Schofield C 1999. The evolution of Chagas disease (American trypanosomiasis) control after 90 years since Carlos Chagas discovery. Mem Inst Oswaldo Cruz 94 (Suppl. 1): 103-121.

Dumonteil E 1999. Update on Chagas' disease in Mexico. Salud Publica Mex 41: 322-327.

Ferreira AW, Moraes de Avila SL 1995. Laboratory diagnosis of Chagas heart disease. São Paulo Med J/RPM 113: 767771

Guzman Bracho C, Garcia Garcia L, Floriani Verdugo J, Guerrero Martinez S, Torres Cosme M, Ramirez Melgar C, Velasco Castrejon O 1998. Risk of transmission of Trypanosoma cruzi by blood transfusion in Mexico. Rev Panam Salud Publica 4: 94-99.

Hernandez-Becerril N, Mejia AM, Ballinas-Verdugo MA, GarzaMurillo V, Manilla-Toquero E, Lopez R, Trevethan S, Cardenas M, Reyes PA, Hirayama K, Monteon VM 2005. Blood transfusion and iatrogenic risks in Mexico City. AntiTrypanosoma cruzi seroprevalence in 43,048 blood donors, evaluation of parasitemia, and electrocardiogram findings in seropositive. Mem Inst Oswaldo Cruz 100: 111-116.

Huante-Magaña R, Piza-Bernal R, Tabárez-Hernández J, LieraRomero F, Mata-Carbajal E, Matadamas N 1990. Enfermedad de Chagas en Guerrero. Reporte de dos casos confirmados con xenodiagnósticos. Salud Publica Mex 32: 320-324.

Kirchhoff LV, Paredes P, Lomeli-Guerrero A, Paredes-Espinoza M, Ron-Guerrero CS, Delgado-Mejia M, Pena-Munoz JG 2006. Transfusion-associated Chagas disease (American trypanosomiasis) in Mexico: implications for transfusion medicine in the United States. Transfusion 46: 298-304.

Luquetti AO, Miles MA, Rassi A, De Rezende JM, De Souza A, Povoa M, Rodriguez L 1986. Trypanosoma cruzi; zymodeme asosociated with acute and chronic Chagas' disease in central Brazil. Trans R Soc Trop Med Hyg 80: 462-470

Maguire JH, Mott KE, Of R, Guimaraes A, Franca JT, Almeida de Sousa J, Ramos NB, Sherlock IA 1982. A three-year follow-up study of infection with $T$. cruzi and electrocardiographic abnormalities in a rural community in northeast Brazil. Am J Trop Med Hyg 31: 42-47.

Mendes RP, Hoshino-Shimizu S, Moura da Silva AM, Mota I, Heredia RA, Luquetti AO, Leser PG 1997. Serological diagnosis of Chagas disease: a potential confirmatory assay using preserved protein antigens of Trypanosoma cruzi. J Clin Microbiol 35: 1829-1834.

Mazzotti L 1940. Dos casos de enfermedad de Chagas en el estado de Oaxaca. Gac Med Mex 70: 417-420.

Mendoza-Gonzalez Jde D, Miranda-Lluck E, Velazco-Castrejon O, Tinoco-Reyna O, Maciel-Pérez Mde J 1995. Chronic Chagas cardiomyopathy. Report of 60 cases. Arch Inst Cardiol Mex 65: 546-550.

Moncayo A 1992. Chagas disease: epidemiology and prospects for interruption of transmission in the Americas. World Health Stat Q 45: 276-279.

Moncayo A 2003. Chagas disease: current epidemiological trends after the interruption of vectorial and trtansfusional transmission in the Southern Cone countries. Mem Inst Oswaldo Cruz 96: 141-144

Monteon VM, Reyes-Lopez PA 1989. Mechanisms of innate and acquired resistance to Trypanosoma cruzi cruzi. Arch Inst Cardiol Mex 59: 529-535.

Monteon VM, Reyes-Lopez PA, Sosa-Palacio A, Leon-Tello G, Martinez-Murguia J, Sosa-Jurado F 2005. Heterogeneous distribution of the prevalence of anti-Trypanosoma cruzi antibodies among blood donors in the State of Puebla, Mexico. Salud Publica Mex 47: 116-125.

Moraes-Souza H, Bordin JO 1996. Strategies for prevention of transfusion-associated Chagas' disease. Transfus Med Rev 10: 161-170.

Oelemann WM, Teixeira MG, Da Costa GV, Borges-Pereira J, De Castro JA, Rodríguez-Coura J, Peralta JM 1998. Evaluation of three comercial enzyme-linked immunosorbent assays for diagnosis of Chagas disease. J Clin Microbiol 36 : 2423-2427.

OPAS-Organización Panamericana de la Salud 1998. La Salud en las Américas, 569 pp.

Perez-Fuentes R, Sanchez-Guillen MC, Gonzalez-Alvarez C, Monteon VM, Reyes PA, Rosales-Encina JL 1998. Humoral nitric oxide levels and antibody immune response of symptomatic and indeterminate Chagas' disease patients to commercial and autochthonous Trypanosoma cruzi antigen. Am J Trop Med Hyg 58: 715-720.

Perez-Fuentes R, Guegan J-F, Barnabe C, Lopez-Colombo A, Salgado-Rosas H, Torres-Rasgado E, Briones B, RomeroDiaz M, Ramos-Jimenez J, Sanchez-Guillen MC 2003. Severity of chronic Chagas disease is associated with cytokine/ antioxidant imbalance in chronically infected individuals. Int J Parasitol 33: 293-299.

Prata A 2001. Clinical and epidemiological aspects of Chagas disease. Lancet Infect Dis 1: 92-100.

Ramos-Echevarria AA, Monteon-Padilla VM, Reyes-Lopez PA 1993. Detection of antibodies against Trypanosoma cruzi in blood donors. Salud Publica Mex 35: 56-64.

Rangel H, Gatica R, Ramos C 1998. Detection of antibodies against Trypanosoma cruzi in donors from a blood bank in Cuernavaca, Morelos, Mexico. Arch Med Res 29: 79-82.

Revollo S, Oury B, Laurent JP, Barnabe C, Quesney V, Carriere V, Noel S, Tibayrenc M 1998. Trypanosoma cruzi: impact of clonal evolution of the parasite on its biological and medical properties. Exp Parasitol 89: 30-39.

Sánchez B, Monteón V, Reyes PA, Espinoza B 2001. Standardization of micro-enzyme-linked immunosorbent assay (ELISA) and western blot for detection of Trypanosoma cruzi antibodies using extracts from mexican strains as antigens. Arch Med Res 32: 382-388

Sanchez-Guillen MC, Barnabe C, Guegan JF, Tibayrenc M, Velasquez-Rojas M, Martinez-Munguia J, Salgado-Rosas H, Torres-Rasgado E, Rosas-Ramirez MI, Perez-Fuentes R 2002. High prevalence anti-Trypanosoma cruzi antibodies, among blood donors in the State of Puebla, a non-endemic area of Mexico. Mem Inst Oswaldo Cruz 97: 947-952. 
Storino R, Milei J 1986. Miocardiopatia Chagasica Cronica. Un Enfoque para el Clínico General, Club de Estudio, Buenos Aires, Argentina.

Tay J, Schenone H, Sánchez JT, Robert L 1992. Estado actual de los conocimientos sobre la enfermedad de Chagas en la Republica Mexicana. Bol Chil Parasitol 47: 43-53.

UNDP/World Bank/WHO 1990. Special Program for Research and Training in Tropical Disease. TDR News, 34.

Velasco-Castrejon O, Guzman-Bracho C 1986. Importante of
Chagas disease in Mexico. Rev Latinoam Microbiol 28: 275283.

Velasco CO, Valdespino JL, Tapia CRC, Salvatierra C, GuzmánBracho C, Magos C, Llausas A, Gutiérrez G, Sepúlveda J 1992. Seroepidemiología de la enfermedad de Chagas en México. Salud Publica Mex 34: 186-196.

WHO-World Health Organization 2002. Control of Chagas disease. Second report of the WHO Expert Committee. WHO Tech Rep Ser 905: 59-90. 